\title{
Can Mixed Parasite Infections Thwart Targeted Malaria Elimination Program in India?
}

\author{
Upasana Shyamsunder Singh, ${ }^{1}$ Nisha Siwal, ${ }^{1}$ Veena Pande, ${ }^{2}$ and Aparup Das ${ }^{1}$ \\ ${ }^{1}$ Division of Genomic Epidemiology, ICMR-Centre for Research in Medical Entomology, No. 4, Sarojini Street, Chinna Chokkikulam, \\ Madurai 625002, India \\ ${ }^{2}$ Department of Biotechnology, Kumaun University, Nainital 263001, India
}

Correspondence should be addressed to Aparup Das; aparupdas@crme.res.in

Received 29 May 2017; Revised 9 July 2017; Accepted 13 July 2017; Published 16 August 2017

Academic Editor: Christophe Duranton

Copyright (C) 2017 Upasana Shyamsunder Singh et al. This is an open access article distributed under the Creative Commons Attribution License, which permits unrestricted use, distribution, and reproduction in any medium, provided the original work is properly cited.

India is highly endemic to malaria with prevalence of all five species of human malaria parasites of Plasmodium genus. India is set for malaria elimination by 2030. Since cases of mixed Plasmodium species infections remain usually undetected but cause huge disease burden, in order to understand the distributional prevalence of both monospecies infections and mixed species infections in India, we collated published data on the differential infection incidences of the five different malaria parasites based on PCR diagnostic assay. About $11 \%$ of total cases were due to mixed species infection. Among several interesting observations on both single and mixed parasitic infections, incidences of Plasmodium falciparum monoinfection were found to be significantly higher than $P$. vivax monoinfection. Also, $P$. malariae seems to be emerging as a potential malaria threat in India. Putting all the facts together, it appears that the dream of achieving malaria elimination in India will not be completely successful without dealing with mixed species infection.

\section{Introduction}

Humans simultaneously infected by different parasites are quite natural and frequent [1]. Around 14,000 pathogens have been described in humans [2] and approximately $30 \%$ of infections may be mixed infections or coinfections, although this can rise up to $80 \%$ in some human populations [3]. By coinfecting a host, parasites form a coevolutionary network [4] which can alter host susceptibility to other parasites, infection duration, transmission risks, and clinical symptoms. This condition poses potential threat to human health in treatment and prevention of the disease. Also, competition may occur between two or more parasite species of the same genus because they occupy the same physical space within their host [5]. In this scenario, gene exchange may take place via recombination or plasmid transfer that could be a potent driver of pathogen evolution, consequently resulting in functional changes which make the parasites highly virulent or resistant to drugs $[6,7]$.
To this extent, malaria is an age-old disease of the human kind, mainly restricted to the tropical and subtropical countries of the globe [8]. Several efforts with basic, applied, and operational research across the globe have not yet been successful in completely controlling malaria, although malaria infection has been severely curtailed by intervention through vector control and chemotherapy [9]. However, evolution and spread of drug-resistant, highly virulent parasites and insecticide-resistant mosquitoes have put new challenges in curtailing malaria to a significant extent [10]. Furthermore, due to large usage of improved surveillance and sensitive diagnosis tools in recent years, it is now easier to detect infections (either with single or with multiple species of human malaria parasites) in a single individual than before [11]. To this extent, the PCR diagnostic assay has evolved over the last decades as the principal and highly sensitive tool for malaria diagnosis in the laboratory [12]. This is because researchers from almost every malaria endemic country have utilized this diagnostic tool to determine the cases of single 
parasitic as well as mixed parasitic infections in a single individual in the laboratory setting.

India is highly endemic to malaria and one of the few countries contributing significantly to global deaths and morbidity due to malaria infection [13]. In 2015, according to estimate by the World Health Organization, about 140 million Indians were infected and there were 384 deaths due to malaria [14]. Varied and changing climatic conditions, urbanisation, global migration, man-made developmental changes, irrigation, and so forth, along with presence of all the five different species of Plasmodium and abundant distribution of nine species of Anopheles that are vectors of malaria parasites, have resulted in bringing out complex malaria in India [15]. Like in many endemic countries, laboratory diagnosis of malaria for epidemiological studies has taken a giant leap from microscopy to PCR diagnostic assay in India. As a result of this, enormous amounts of data on monoparasitic infections and mixed parasitic infections have been accumulated over the past decade across different malaria endemic provinces of India. Considering India is aiming to eliminate malaria by 2030 [16], as a first step to achieve this goal, it is of importance to understand abundance and differential distribution by both monoinfections and mixed infections of five different species of malaria parasites infecting human (Plasmodium falciparum, $P$. vivax, $P$. malariae, $P$. ovale, and $P$. knowlesi). Therefore, (i) in order to determine distributional abundance of mono-malaria parasite infections as well as mixed malaria parasite infections in all of India in a single communication, (ii) to generate debate on the probable cause of differential interactions of two or more different species of Plasmodium infecting a single individual at a time, and (iii) to synthesize if the current knowledge would be useful for the targeted malaria elimination program in India, we have collated published data (published between 2005 and 2017) on mono-malaria infections and mixed malaria infections based on PCR diagnosis in India [17-34] and present herewith comprehensive information that could be of potential interest to malaria public health in terms of targeted malaria elimination in India.

\section{Uprising of $P$. falciparum, Range Expansion of $P$. malariae, and Abundance of Mixed Species Malaria Infection}

Collation of data from 19 publications involving 7821 malaria positive individuals sampled in 67 different geographic locations of 18 different Indian states at different time points (Table 1) reveals several interesting findings. If only the burden of infections by five different malaria parasites is considered (irrespective of their single or mixed infections), majorities were found to be due to P. falciparum (64\%), followed by $P$. vivax (33\%). The other three species have meagre contribution ( $P$. malariae, 2\%; $P$. knowlesi, $0.9 \%$; and $P$. ovale $0.1 \%$ ) to malaria infection in India (Figure $1(\mathrm{a})$ ). If the extent of monoinfection across different malaria endemic Indian states is considered, $P$. falciparum was highest in Chhattisgarh (87\%) followed by Goa (79\%) and lowest in Tamil Nadu
(20\%) (Figure 1(b)). Similarly, highest monoinfection by $P$. vivax was evident in Delhi (66\%) followed by Uttar Pradesh (62\%) and the lowest one was in Chhattisgarh (0.33\%) with no cases in Jharkhand (Figure 1(b)). Interestingly, monoinfection by $P$. malariae could be detected in five states with highest abundance in Odisha (6.4\%) followed by Tamil Nadu (5.3\%), Andhra Pradesh (3.3\%), Madhya Pradesh (1\%), and Asom $(0.7 \%)$. However, monoinfections of $P$. ovale were found only in two individuals from Chhattisgarh, and P. knowlesi was restricted to Andaman and Nicobar only in three cases (Figure 1(b)). It is therefore evident that monoinfections due to $P$. falciparum have surpassed $P$. vivax in India. About a decade ago, the proportion of $P$. falciparum to $P$. vivax was about $50: 50$ [15], but at present this turns out to be $73: 27$. To be noted that Indian $P$. falciparum is resistant to chloroquine and other sulpha drugs [35]. Furthermore, large number of asymptomatic $P$. falciparum infections circulating in local populations in different endemic states of India [36, 37] and failure in efficacy of the most effective and dependable antimalarial, ACT (artemisinin combined therapy) have been reported in several Indian locations [38, 39]. Therefore, management of rising monoinfection by $P$. falciparum in India seems to be quite an uphill task. Considering alone the upsurge of $P$. falciparum monoinfection, the situation is quite alarming in terms of malaria public health in India.

Malaria complexity in India is further compounded by the fact that all the five species of Plasmodium are often found to be in coinfection in a single individual. When data on mixed infections (in any combination among five different species) in a single individual are segregated based on different states, the incidence of mixed infection was found to be highest in Karnataka (30\%), followed by Jharkhand (27\%), Odisha (23\%), Andhra Pradesh (18.5\%), Andaman and Nicobar Islands (17.7\%), Tamil Nadu (17\%), North-eastern states (16.4\%), Maharashtra (14\%), Chhattisgarh (11.8\%), Gujarat and Rajasthan (9\%), Uttar Pradesh (5.6\%), Madhya Pradesh (4.3\%), Delhi (2.9\%) and Goa (2.6\%), with no cases of mixed infection in Bihar (Figure 1(b)). It is interesting to note that states presenting less number of monoinfection by $P$. falciparum (e.g., Karnataka) had the highest cases of mixed infection due to $P$. vivax and $P$. falciparum. Similarly, in Jharkhand where not a single case of $P$. vivax monoinfection was detected, $27 \%$ of mixed infections are due to $P$. vivax and $P$. falciparum coinfection. Apart from the mixed infection of $P$. falciparum and $P$. vivax, coinfections among other species of Plasmodium in India are quite infrequent with an exception of mixed infection involving $P$. ovale with either $P$. vivax or $P$. knowlesi in India. Among all mixed infection cases, one such case (between $P$. malariae and $P$. falciparum) needs significant attention. Mixed infection due to these two species was found to be distributed across several Indian locations, especially in the eastern and western parts (Figure 2(a)). P. malariae was once prevalent only in the eastern part of India (e.g., Odisha [40]) but has now expanded its range to the west also, that is, in Rajasthan and Gujarat (Figure 2(a)). This is possibly due to the fact that $P$. malariae can coinfect with $P$. vivax and $P$. ovale, though to a lesser extent than with $P$. falciparum. Since $P$. vivax is a well-adapted malaria parasite in India and endemic across Indian localities, the range expansion 
TABLE 1: Details of data on the differential prevalence of monoinfections and mixed infections by PCR diagnostic assay in India. To be noted is that the dataset consists of 67 different locations taken from 18 Indian states, retrieved from 19 different publications spanning from 2005 to 2017.

\begin{tabular}{|c|c|c|c|}
\hline $\begin{array}{l}\text { Sr. } \\
\text { number }\end{array}$ & Name of state & $\begin{array}{l}\text { Location and year (in parenthesis) of } \\
\text { sample collection }\end{array}$ & $\begin{array}{c}\text { Reference and number (in } \\
\text { parenthesis) }\end{array}$ \\
\hline \multirow{3}{*}{ (1) } & \multirow{3}{*}{ Delhi } & $\begin{array}{l}\text { Nandnagri (2004) } \\
\text { Shahdara }(2004)\end{array}$ & Nandwani et al. 2005 [17] \\
\hline & & Dwarka (2011-2013) & Singh et al. 2014 [18] \\
\hline & & Delhi (2014) & Unpublished data \\
\hline \multirow{9}{*}{ (2) } & \multirow{9}{*}{ Asom } & Asom (2004-2006) & Keen et al. 2007 [19] \\
\hline & & Sonapur (2007) & Gupta et al. 2010 [20] \\
\hline & & Tezpur (2011-2012) & Haanshuus et al. 2016 [21] \\
\hline & & Diphu (2013) & Unpublished data \\
\hline & & Guwahati (2013) & Unpublished data \\
\hline & & Dibrugarh (2011-2012) & \multirow{4}{*}{ Sharma et al. 2013 [22] } \\
\hline & & Tinsukia (2011-2012) & \\
\hline & & Karbi-Anglong (2011-2012) & \\
\hline & & Chirang (2011-2012) & \\
\hline \multirow{2}{*}{ (3) } & Arunachal & Rohit (2011-2012) & \multirow{2}{*}{ Sharma et al. 2013 [22] } \\
\hline & Pradesh & Changlang (2011-2012) & \\
\hline \multirow{2}{*}{ (4) } & \multirow{2}{*}{ Tripura } & Manu Bazar (2014) & Krishna et al. 2015 [23] \\
\hline & & Shantir Bazar (2014) & Krishna et al. 2015 [23] \\
\hline (5) & Uttar Pradesh & Shankargarh (2015) & Unpublished data \\
\hline \multirow{3}{*}{ (6) } & \multirow{3}{*}{ Rajasthan } & Bikaner (2007-2008) & Kochar et al. 2014 [24] \\
\hline & & Bikaner (2010-2011) & Nayak et al. 2013 [25] \\
\hline & & Udaipur (2014) & Krishna et al. 2015 [23] \\
\hline (7) & Bihar & Raxaul (2011-2012) & Haanshuus et al. 2016 [21] \\
\hline \multirow{10}{*}{ (8) } & \multirow{10}{*}{ Madhya Pradesh } & Madhya Pradesh (2004-2006) & Keen et al. 2007 [19] \\
\hline & & Shivpuri (2009) & \\
\hline & & Dindori (2009) & Singh et al. 2010 [26] \\
\hline & & Balaghat (2010-2012) & Patel et al 2014 [27] \\
\hline & & Jabalpur (2010-2012) & Patel et al. 2014 [27] \\
\hline & & Jabalpur (2008-2012) & Jain et al. 2013 [28] \\
\hline & & Balaghat (2012) & Bharti et al. 2013 [29] \\
\hline & & Betul (2012-2013) & Unpublished data \\
\hline & & Jhabua (2014) & Krishna et al. 2015 [23] \\
\hline & & Annupur (2014) & Krishna et al. 2015 [23] \\
\hline \multirow{2}{*}{ (9) } & \multirow{2}{*}{ Jharkhand } & Jaldega (2014) & Krishna et al. 2015 [23] \\
\hline & & Bano (2014) & Krishna et al. 2015 [23] \\
\hline \multirow{3}{*}{$(10)$} & \multirow{3}{*}{ Gujarat } & Dahod (2014) & Krishna et al. 2015 [23] \\
\hline & & Valsad (2014) & Krishna et al. 2015 [23] \\
\hline & & Nadiad (2015) & Unpublished data \\
\hline \multirow{6}{*}{ (11) } & \multirow{6}{*}{ Chhattisgarh } & Raipur (2007) & Gupta et al. 2010 [20] \\
\hline & & Bilaspur (2010) & Kumar et al. 2013 [32] \\
\hline & & Mungeli (2011-2012) & Haanshuus et al. 2016 [21] \\
\hline & & Jagdalpur (2014) & Krishna et al. 2015 [23] \\
\hline & & Baikunthpur (2014) & Krishna et al. 2015 [23] \\
\hline & & Bastar (2013-2014) & Chaturvedi et al. 2015 [30] \\
\hline \multirow{5}{*}{ (12) } & \multirow{5}{*}{ Maharashtra } & Maharashtra (2004-2006) & Keen et al. 2007 [19] \\
\hline & & Ratnagiri (2011-12) & Haanshuus et al. 2016 [21] \\
\hline & & Gadchiroli (2012) & Unpublished data \\
\hline & & Gadchiroli (2014) & Krishna et al. 2015 [23] \\
\hline & & Gondia (2014) & Krishna et al. 2015 [23] \\
\hline
\end{tabular}


TABle 1: Continued.

\begin{tabular}{|c|c|c|c|}
\hline $\begin{array}{l}\text { Sr. } \\
\text { number }\end{array}$ & Name of state & $\begin{array}{l}\text { Location and year (in parenthesis) of } \\
\text { sample collection }\end{array}$ & $\begin{array}{c}\text { Reference and number (in } \\
\text { parenthesis) }\end{array}$ \\
\hline \multirow{16}{*}{ (13) } & \multirow{16}{*}{ Odisha } & Odisha (2004-2006) & Keen et al. 2007 [19] \\
\hline & & Rourkela (2008) & Gupta et al. 2010 [20] \\
\hline & & Mayurbhanj (2008) & \\
\hline & & Sundergarh (2008) & \\
\hline & & Keonjhar (2008) & \\
\hline & & Nayagarh (2008) & \\
\hline & & Rayagada (2008) & Dhangadamajh1 et al. 2009 [31] \\
\hline & & Kalahandi (2008) & \\
\hline & & Kandhamal (2008) & \multirow{4}{*}{ Pati et al. (2017) [34] } \\
\hline & & Anugul (2008) & \\
\hline & & Keonjhar (2012-2014) & \\
\hline & & Mayurbhanj (2012-2014) & \\
\hline & & Keonjhar (2013) & Unpublished data \\
\hline & & Rourkela (2012) & Unpublished data \\
\hline & & Koraput (2014) & Krishna et al. 2015 [23] \\
\hline & & Rayagada (2014) & Krishna et al. 2015 [23] \\
\hline (14) & Andhra Pradesh & Anantapur (2011-12) & Haanshuus et al. 2016 [21] \\
\hline \multirow{2}{*}{ (15) } & \multirow{2}{*}{ Goa } & Goa (2004-06) & Keen et al. 2007 [19] \\
\hline & & Panaji (2008) & Gupta et al. 2010 [20] \\
\hline \multirow{2}{*}{ (16) } & \multirow{2}{*}{ Karnataka } & Bengaluru (2008) & Gupta et al. 2010 [20] \\
\hline & & Mangalore (2014) & Unpublished data \\
\hline \multirow{4}{*}{ (17) } & \multirow{4}{*}{ Tamil Nadu } & Chennai (2008) & Gupta et al. 2010 [20] \\
\hline & & Oddanchatram (2011-2012) & Haanshuus et al. 2016 [21] \\
\hline & & Ambur (2011-12) & Haanshuus et al. 2016 [21] \\
\hline & & Chennai (2014) & Unpublished data \\
\hline (18) & $\begin{array}{l}\text { Andaman \& } \\
\text { Nicobar }\end{array}$ & $\begin{array}{c}\text { PortBlair (2004) } \\
\text { Car Nicobar (2004) } \\
\text { Teressa }(2009) \\
\text { Campbell Bay (2010) }\end{array}$ & Tyagi et al. 2013 [33] \\
\hline
\end{tabular}

of P. malariae might have happened due to its ability to coinfect with $P$. vivax in India. Similarly, mixed infection due to $P$. ovale and $P$. falciparum which was reported in Odisha [41] has now expanded its range to central part of Indian states like Madhya Pradesh, Chhattisgarh, and Jharkhand (Figure 2(b)). It is interesting to note that all the diversities of mixed species infection of malaria parasites in possible combinations involving $P$. falciparum, $P$. ovale, $P$. vivax, and $P$. malariae are present in Odisha (Figures 2(a) and 2(b)). To be noted here is that both $P$. malariae and $P$. ovale are highly prevalent in the Thai-Myanmar border and in other Southeast Asian countries which are almost at the same latitude as that of Odisha [42-44]. Furthermore, Odisha had historical trade practice with Southeast Asian countries in the recent past. Therefore, observed similarities in patterns of mixed malaria parasite infections in Odisha and Southeast Asian countries are not surprising.

In order to understand if any defined trend of association exists in the occurrence of different monoinfections and mixed infections, it was interesting to note that $P$. vivax monoinfections was statistically significantly positively correlated ( $r=0.8867 ; P<0.00001)$ to $P$. falciparum across 18
Indian states. This indicates that Indian populations are susceptible for infection by both the parasite species and therefore explains the unique burden of both $P$. vivax and $P$. falciparum in India. This situation is opposite to Vanuatu where negative correlation in occurrence of these two species had been reported [45]. This situation is reflected by the fact that $P$. falciparum and $P$. vivax have different origins and they are placed far in phylogenetic tree as compared to the other human malaria parasites [46]. Interestingly, monoinfections of both the species were also found to be statistically significantly positively correlated to the mixed infection by both the species across Indian populations ( $P$. falciparum monoinfection versus $P$. falciparum $+P$. vivax, $r=0.6646, P<0.004$; $P$. vivax monoinfection versus $P$. vivax $+P$. falciparum, $r=$ $0.6722, P<0.003)$. This means that mixed species infections are prevalent in both $P$. vivax endemic and $P$. falciparum endemic localities in India, thereby justifying that both of these parasite species have evolved greatly to coinfect Indians. Incidentally, both monoinfections and mixed infections by $P$. malariae were statistically strongly positively correlated with different combinations of species ( $P$. malariae versus $P$. falciparum $+P$. malariae, $r=0.9537, P<0.00001 ;$. malariae 


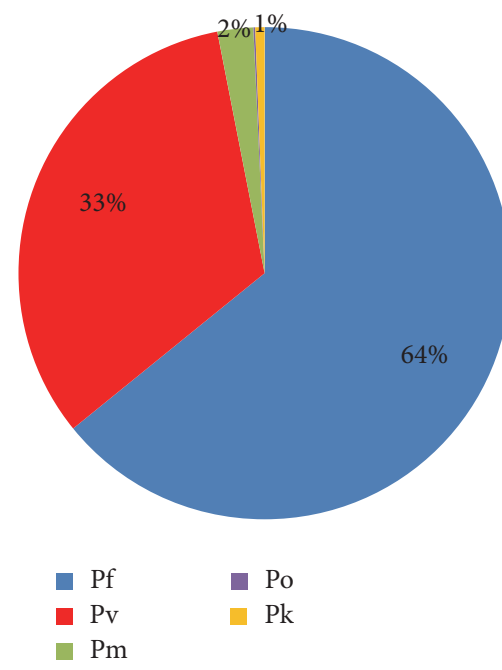

(a)

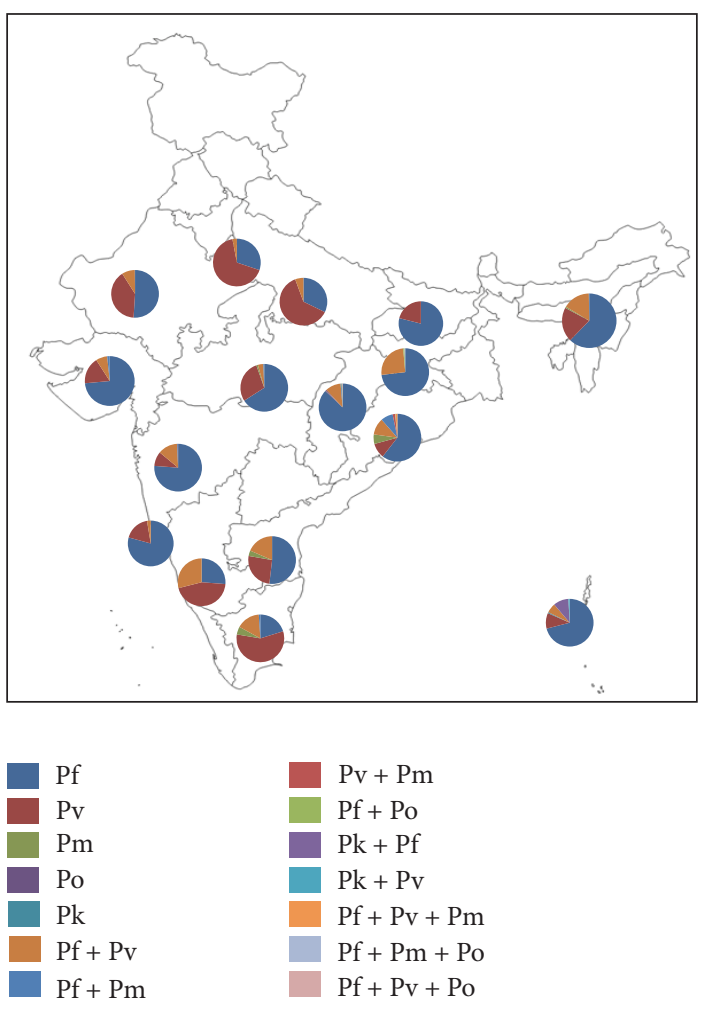

(b)

FIGURE 1: Malaria burden due to infection of different malaria parasites either as single or as mixed infection in India. (a) Pie chart showing contribution of each of the five human malaria parasite species (both monospecies infections and mixed species infections have been considered) into the net malaria burden in India. Clearly, P. falciparum has surpassed P. vivax and P. malariae is increasing its abundance in India. (b) Pie chart of infections with either mono-malaria parasites or different combinations of mixed malaria parasites in different Indian states (keys of different colour codes are given in the figure legend).

versus $P$. vivax $+P$. malariae, $r=0.8559, P<0.0002 ; P$. falciparum $+P$. malariae versus $P$. vivax $+P$. malariae, $r=$ $0.9684, P<0.00001)$. This indicates the adaptive affinity of $P$. malariae to coinfect with every other species of Plasmodium in a single individual in India. Interestingly, very few cases of coinfection involving $P$. ovale and $P$. knowlesi with other species could be found indicating their poor adaptive capacity in a coinfection state in a single human host in India.

\section{Mixed Malaria Infection: Adaptive Evolution Favouring Parasitism?}

The variable patterns of coinfection of five different species of the human malaria parasites in India (Figure 3) need to be discussed in terms of (i) preferential invasion of human Red Blood Cells (RBCs) of five different parasite species and (ii) intrahost competitive abilities. Firstly, the invasion preferences developed by human Plasmodium parasites have evolved to create a favourable environment for the parasites that provides abundant opportunity for transmission (Figure 4). It is often argued that degree of evolution in self-limiting techniques, such as preferential RBC invasion, is linked to the degree of concordant evolution of the parasite within its host [47]. Furthermore, wherever there is a strong preferential invasion of young RBCs, the maximum parasite replication rate has little impact on acute and chronic anaemia [47]. This is exemplified by the fact that $P$. falciparum has no selection for the type of RBCs for invasion (it invades both the young and matured RBCs, alike) (Figure 4), and that might be the reason for severe manifestation. On the other hand, $P$. vivax, $P$. ovale, and $P$. malariae, being selective for the type of RBC (Figure 4), cause less severe malaria [47]. Furthermore, preference of invasion of human reticulocytes by $P$. knowlesi merozoites has been suggested [48]. Competition may occur between two parasite species because they occupy the same physical space within their host [5]. Accordingly, P. ovale and P. vivax merozoites have similar preference for reticulocytes [47], which is why probably they do not coexist. If the parasites modify their respective ecological niches, they might in due course be able to coexist [49] and such cellular niche expansion may increase adaptive parasitism, which, in turn, may also increase pathogenesis. For example, it has been shown in vitro that $P$. knowlesi, which otherwise invaded all kinds of RBCs of its natural host macaque, can also invade a wider age range of human RBCs [50]. Considering the smallest lifecycle of only 24 hours of this species among all other species infecting human, if P. knowlesi evolves to invade all kinds of human RBCs, it might emerge as the most fatal of all the Plasmodium species infecting humans. 


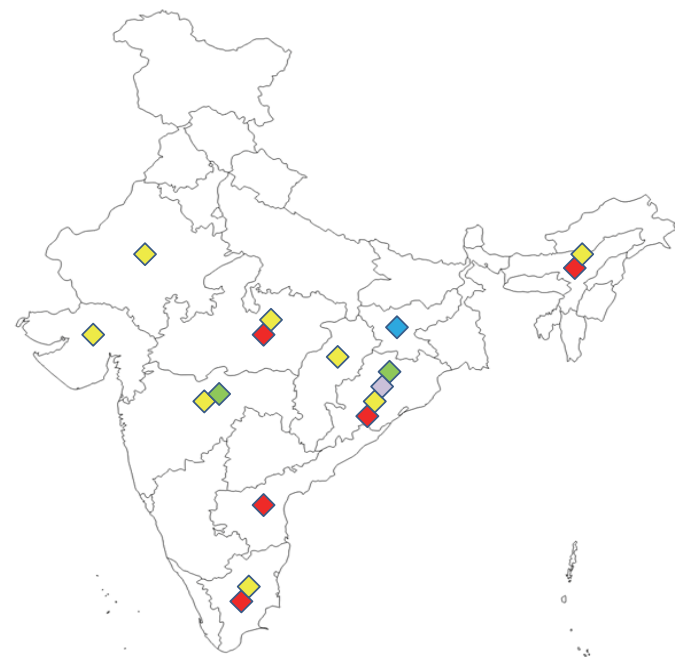

P. malarie

P. vivax + P. malarie

$\diamond$ P. falciparum + P. malarie $\checkmark$ P. falciparum + P. vivax + P. malarie P. falciparum + P. ovale + P. malarie

(a)

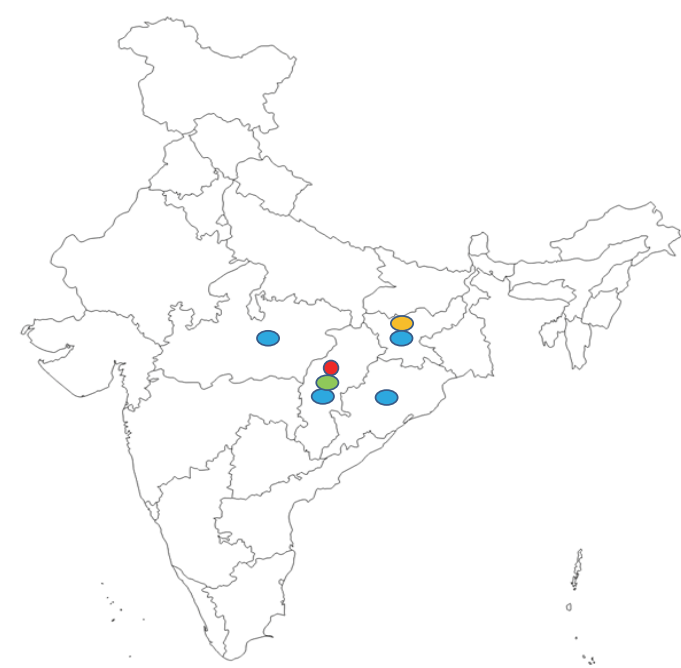

- P. ovale

- P. falciparum + P. ovale

P. falciparum + P. ovale + P. malarie

- P. falciparum + P. ovale + P. vivax

(b)

Figure 2: Distributional prevalence of the two less prevalent malaria parasites ( $P$. malariae and $P$. ovale) in India. (a) To be noted is that $P$. malariae has been able to expand its range in almost all endemic locations in India, primarily as mixed infection with other species and principally in coinfection with $P$. falciparum. Odisha serves as the epicentre of every combination of diversity of coinfection by $P$. malariae with four other species of Plasmodium in India. (b) In contrast to P. malariae, P. ovale still has a very limited distribution, its capacity to coinfect with $P$. falciparum has helped in expanding its range in four different Indian states.

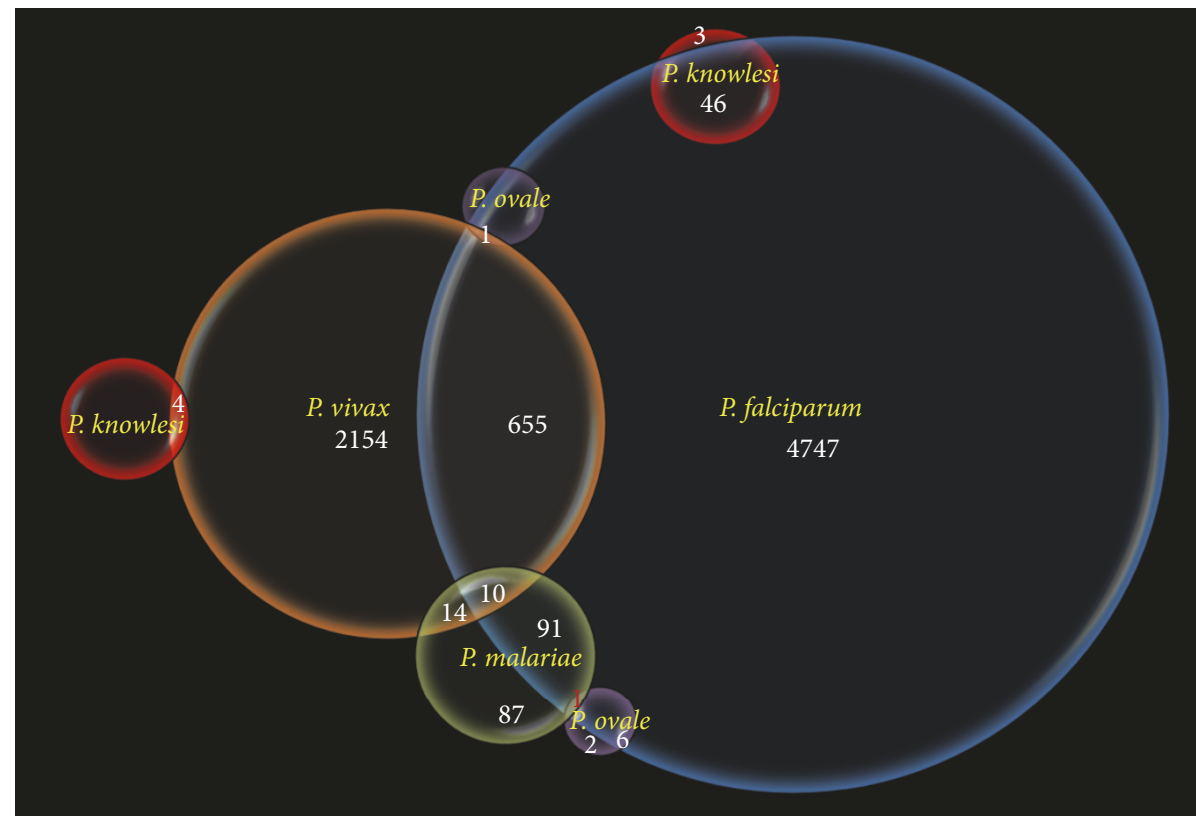

FIGURE 3: Venn diagram showing differential incidences and amount of interactions among five different species of malaria parasites in India. The numbers indicated against the name of the Plasmodium species are actual numbers of monoinfections and those that are indicated at each interaction are the number of mixed infections. Each circle is represented by a single species of Plasmodium and the size of the circles represents the relative prevalence of that species in India as evidenced through PCR diagnosis. To be noted here is that $P$. falciparum infections have grossly surpassed P. vivax infections in India. P. knowlesi, restricted so far to the Andaman and Nicobar Islands, is principally found as mixed infection either with $P$. vivax or with $P$. falciparum. Likewise, $P$. malariae is principally found as coinfection, that too majorly with $P$. falciparum. 


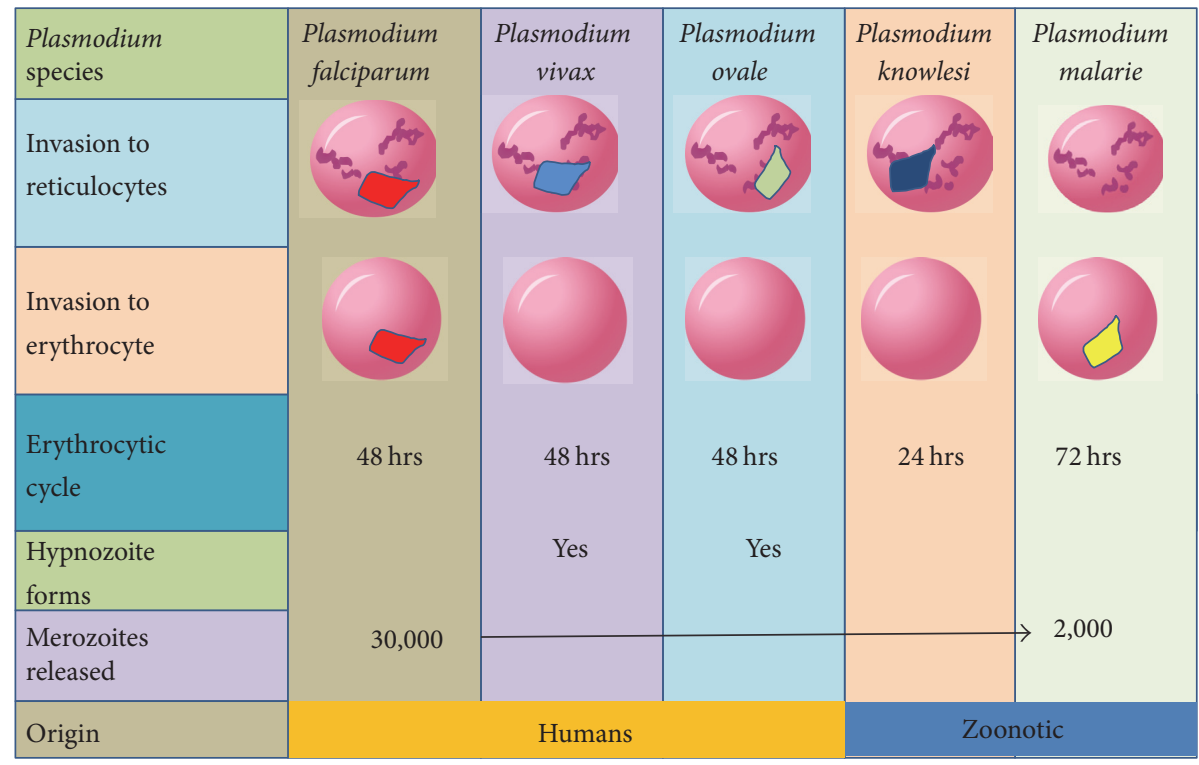

Figure 4: Host preference to different types of human Red Blood Cells (RBCs) by five different malaria parasites. To be noted is that, based on the common-niche-high-competition hypotheses, more incidences of mixed malaria parasitic infections were found in species that share dissimilar ecological niche (types of human RBCs).

In a genetic viewpoint, $P$. ovale and $P$. vivax have similar breadth and sequence type of the pir (Plasmodium interspersed repeats) gene repertoires [51], which is the largest multigene family present in all Plasmodium genomes reported thus far [52]. Although the function of this protein family is not completely understood, it seems to promote parasite development in both the liver and blood, either by supporting parasite development within hepatocytes and erythrocytes and/or by manipulating the host immune response [53]. Since $P$. malariae contains only a restricted subset of the pir gene family [51] and almost 50\% of pir genes in $P$. malariae are pseudogenes, host adaptation in $P$. malariae is less restricted than in $P$. falciparum [51]. This might probably explain why $P$. malariae is mostly found as mixed infection with other species of Plasmodium in India (Figure 3). Also, the kelch13 gene of $P$. falciparum (associated with artemisinin resistance) is $84.8 \%$ and $82.7 \%$ similar to $P$. malariae and $P$. ovale, respectively [54]. Moreover, in the present dataset, P. knowlesi shows its prevalence more as a coinfection with $P$. falciparum than infecting as a single parasite. Since the three species ( $P$. knowlesi, $P$. malariae, and $P$. ovale) are phylogenetically close to each other [55] and are also selective for specific types of RBC (Figure 4), it might be true that these three species of Plasmodium are in the learning phase and therefore seek help of an evolved parasite $(P$. falciparum) that can invade any kind of RBC (Figure 4) [47]. Therefore, malaria parasites are continuously evolving for better parasitism through their less-complex genomes [56] and less generation time, in comparison to their human or mosquito hosts. For example, considering the possibilities for regular genetic exchange within $P$. malariae populations [57], development of a selective inhibitor for $P$. malariae may prove more challenging than the development of one for $P$. falciparum [58].
Adaptation by Darwinian natural selection at both genetic and phenotypic level is evidenced in many species including malaria parasites in India [59]. Cases of nonrandom association between different parasites coinfecting a single host is also an indication of preferential (and therefore adaptive in nature) association. Some such incidences are worth mentioning here. For example, $P$. ovale was invariably found in association with $P$. falciparum and $P$. malariae, and not a single case of $P$. ovale to coinfect with $P$. knowlesi and $P$. vivax could be detected. This can be explained in terms of the fact that both $P$. vivax and $P$. ovale form hypnozoites (dormant parasite stages in the liver that cause relapse weeks to years after the primary infection [60]) therefore share a common niche. It is widely known that niche sharing leads to competition; thus, in order to avoid intrahost competition, $P$. ovale might not associate with $P$. vivax in a coinfection. The present epidemiological finding in India together with similar finding in other malaria endemic countries on coexistence of five different species at different combinations (Canada, $P$. ovale and P. falciparum [61]; China-Myanmar border, $P$. malariae and $P$. ovale [44]; Switzerland, $P$. falciparum and $P$. malariae [62]; and China, P. knowlesi and P. falciparum [63]) on the differential species infection in a single host can be discussed by the "hitchhiking" hypothesis [64]. Accordingly, when two different species coinfect a single host, one parasite species is unable to manipulate the host machinery, getting benefit from the manipulative potential of the other species [64]. The epidemiological finding on the coinfection of five different malaria parasites in India thus seems to follow the "hitchhiking" hypothesis, as the three otherwise less prevalent malaria parasites ( $P$. malariae, $P$. knowlesi and $P$. ovale) mostly occur as coinfections mainly with $P$. falciparum and to certain extent with $P$. vivax. 


\section{Conclusions and Future Prospects}

Clearly, India is heavily burdened with appreciable number of malaria cases with increasing number of $P$. falciparum monoinfections, and mixed Plasmodium species infections in a single individual. However, conclusions made out of the results presented in this communications suffer from several limitations. For example, (i) the PCR diagnostic assay has been performed in many different laboratories without following a uniform molecular biological protocol, (ii) the results of mixed infection came as a by-product of some other research work, and therefore sampling might have been biased, (iii) malaria samples have not been collected in defined period of year, and (iv) data from each and every malaria endemic locations have been included in the present analysis. Even then, the present dataset, prepared out of collation of published literature on mixed malaria infection, provides enough insight on the trends of increasing incidences of $P$. falciparum monoinfection and occurrence of mixed infection of five different malaria parasites in variable proportions.

Over several years, many agendas (either global or local) for either eradication or elimination of malaria have been prepared and implemented. The Global Malaria Eradication Program (GMEP), which ran from 1955 to 1969, had helped in reduction of malaria cases globally to a significant extent. However, technical challenges and resurgence of malaria in many countries including India led to a shift away from malaria elimination for many decades. Since 2000, there has been an increasing political drive to eliminate malaria [65], and reducing the burden of malaria is a central component of the Millennium Development Goals (MDGs) [65]. The current approaches to malaria elimination by the World Health Organization (WHO) have been fueled by the GMEP, which can crown a country to have achieved malaria elimination when there is reduction to zero of the incidence of infection caused by a specified malaria parasite in a defined geographical area as a result of deliberate efforts [66]. With a view to eliminate malaria in India, a national framework for malaria elimination in India (2016-2030) has been prepared which aims to eliminate malaria (zero indigenous cases) in the entire country by 2030. Accordingly, it is planned to eliminate malaria from 15 low-endemic states and Union Territories by 2020 and from moderate endemic states by 2022 and ultimately eliminating malaria from the whole country by 2027 and sustain zero transmission until the next three years to be officially declared by the WHO as a malaria-free country [67]. However, neither nationwide epidemiological information nor disease burden due to mixed species infection is available in India. The information presented here in one way throws enough light on malaria burden due to increasing $P$. falciparum monoinfection as well as mixed species infection and entails considerable risks that might come in the way of ambitious malaria elimination program in India. Considering (i) the fact that there is no defined diagnostic method for detection of mixed species infection at the local health centre, (ii) high species diversity of anopheline mosquitoes that are vectors to Plasmodium parasites, and (iii) unavailability of a defined treatment program for mixed species infection and increasing trends of drug-resistant parasites [68], goals set for malaria elimination seem far from achievement in India. Integrated efforts with in-depth surveillance for (i) malaria epidemiology specially focussing on increasing incidences of $P$. falciparum malaria and expanding range of $P$. malariae, (ii) type of infection (monospecies/mixed species), and (iii) distribution of drug and insecticide resistance malaria parasites and vectors, respectively $[69,70]$, would provide baseline data on which malaria elimination strategy could be assembled with effective case management and parallel control of local mosquito vectors. As of now, malaria elimination in India seems to be quite an uphill task.

\section{Disclosure}

Upasana Shyamsunder Singh is a Research Assistant; Nisha Siwal is a Senior Research Fellow.

\section{Conflicts of Interest}

The authors declare no conflicts of interest.

\section{Authors' Contributions}

Upasana Shyamsunder Singh and Nisha Siwal have collected data from published literature. Upasana Shyamsunder Singh had performed data analyses, prepared figures and initial version of the manuscript. Veena Pande has helped in data analyses. Aparup Das has conceptualized and designed the study, interpreted data, and prepared the final version of the manuscript.

\section{Acknowledgments}

The authors thank their colleagues who have put their effort in generating data on mixed malaria species infection in India on the basis of which this article has been generated. They thank the Indian Council of Medical Research for intramural funding assistance.

\section{References}

[1] T. Rigaud, M.-J. Perrot-Minnot, and M. J. F. Brown, "Parasite and host assemblages: Embracing the reality will improve our knowledge of parasite transmission and virulence," Proceedings of the Royal Society B: Biological Sciences, vol. 277, no. 1701, pp. 3693-3702, 2010.

[2] L. H. Taylor, S. M. Latham, and M. E. J. Woolhouse, "Risk factors for human disease emergence," Philosophical Transactions of the Royal Society B: Biological Sciences, vol. 356, no. 1411, pp. 983989, 2001.

[3] T. N. Petney and R. H. Andrews, "Multiparasite communities in animals and humans: frequency, structure and pathogenic significance," International Journal for Parasitology, vol. 28, no. 3, pp. 377-393, 1998.

[4] M. A. Vannier-Santos and H. L. Lenzi, "Parasites or cohabitants: Cruel omnipresent usurpers or creative "éminences Grises" ?" Journal of Parasitology Research, vol. 2011, Article ID 214174, 19 pages, 2011.

[5] L. Stancampiano, L. M. Gras, and G. Poglayen, "Spatial niche competition among helminth parasites in horse's large intestine," Veterinary Parasitology, vol. 170, no. 1-2, pp. 88-95, 2010. 
[6] G. Dantas and M. O. Sommer, "Context matters - the complex interplay between resistome genotypes and resistance phenotypes," Current Opinion in Microbiology, vol. 15, no. 5, pp. 577582, 2012.

[7] J. A. Perry and G. D. Wright, "The antibiotic resistance "mobilome": Searching for the link between environment and clinic," Frontiers in Microbiology, vol. 4, no. 138, 2013.

[8] S. Plianbangchang, "Indian Society for Malaria and Other Communicable Diseases "Honorary Fellowship" address by Dr Samlee Plianbangchang, Regional Director, World Health Organizaion, South East Asia Region.” The Journal of communicable diseases, vol. 40, no. 1, pp. 1-4, 2008.

[9] J. Gyapong and B. Boatin, "Neglected tropical diseases - subSaharan Africa," Neglected Tropical Diseases, vol. 2016, pp. 87112, 2016.

[10] A. F. Read, P. A. Lynch, and M. B. Thomas, "How to make evolution-proof insecticides for malaria control," PLoS Biology, vol. 7, no. 4, Article ID e1000058, 2009.

[11] G. Snounou, S. Viriyakosol, W. Jarra, S. Thaithong, and K. N. Brown, "Identification of the four human malaria parasite species in field samples by the polymerase chain reaction and detection of a high prevalence of mixed infections," Molecular and Biochemical Parasitology, vol. 58, no. 2, pp. 283-292, 1993.

[12] M. Hawkes and K. C. Kain, "Advances in malaria diagnosis," Expert Review of Anti-Infective Therapy, vol. 5, no. 3, pp. 485495, 2007.

[13] A. Das, A. R. Anvikar, L. J. Cator et al., "Malaria in India: The Center for the Study of Complex Malaria in India," Acta Tropica, vol. 121, no. 3, pp. 267-273, 2012.

[14] World Health Organization (WHO), "World Malaria Report," 2016, http://www.who.int/malaria/publications/world-malariareport-2016/report/en/.

[15] V. Singh, N. Mishra, G. Awasthi, A. P. Dash, and A. Das, "Why is it important to study malaria epidemiology in India?" Trends in Parasitology, vol. 25, no. 10, pp. 452-457, 2009.

[16] G. Newby, A. Bennett, E. Larson et al., "The path to eradication: A progress report on the malaria-eliminating countries," The Lancet, vol. 387, no. 10029, pp. 1775-1784, 2016.

[17] S. Nandwani, M. Mathur, and S. Rawat, "Evaluation of the polymerase chain reaction analysis for diagnosis of falciparum malaria in Delhi, India," Indian Journal of Medical Microbiology, vol. 23, no. 3, pp. 176-178, 2005.

[18] R. Singh, D. P. Singh, R. Gupta et al., "Comparison of three PCRbased assays for the non-invasive diagnosis of malaria: Detection of Plasmodium parasites in blood and saliva," European Journal of Clinical Microbiology and Infectious Diseases, vol. 33, no. 9, pp. 1631-1639, 2014.

[19] J. Keen, G. A. Farcas, K. Zhong, S. Yohanna, M. W. Dunne, and K. C. Kain, "Real-time PCR assay for rapid detection and analysis of PfCRT haplotypes of chloroquine-resistant Plasmodium falciparum isolates from India," Journal of Clinical Microbiology, vol. 45, no. 9, pp. 2889-2893, 2007.

[20] B. Gupta, P. Gupta, A. Sharma, V. Singh, A. P. Dash, and A. Das, "High proportion of mixed-species Plasmodium infections in India revealed by PCR diagnostic assay," Tropical Medicine and International Health, vol. 15, no. 7, pp. 819-824, 2010.

[21] C. G. Haanshuus, S. Chandy, A. Manoharan et al., "A high malaria prevalence identified by PCR among patients with acute undifferentiated fever in India," PLoS ONE, vol. 11, no. 7, Article ID e0158816, 2016.
[22] J. Sharma, P. Dutta, S. A. Khan, P. Chowdhury, J. Borah, and J. Mahanta, "Comparison of different diagnostic techniques for detection of Malaria infection in blood samples collected from Malaria endemic areas of Assam and Arunachal Pradesh," International Journal of Scientific and Engineering Research, vol. 4, no. 9, pp. 2587-2603, 2013.

[23] S. Krishna, P. K. Bharti, H. S. Chandel et al., "Detection of mixed infections with plasmodium spp. by PCR, India, 2014," Emerging Infectious Diseases, vol. 21, no. 10, pp. 1853-1857, 2015.

[24] D. K. Kochar, A. Das, A. Kochar et al., "A prospective study on adult patients of severe malaria caused by Plasmodium falciparum, Plasmodium vivax and mixed infection from Bikaner, northwest India," Journal of Vector Borne Diseases, vol. 51, no. 3, pp. 200-210, 2014.

[25] K. C. Nayak, S. L. Meena, B. K. Gupta, S. Kumar, and V. Pareek, "Cardiovascular involvement in severe vivax and falciparum malaria," Journal of Vector Borne Diseases, vol. 50, no. 4, pp. 285291, 2013.

[26] N. Singh, M. M. Shukla, M. K. Shukla et al., "Field and laboratory comparative evaluation of rapid malaria diagnostic tests versus traditional and molecular techniques in India," Malaria Journal, vol. 9, no. 1, pp. 191-203, 2010.

[27] J. C. Patel, N. W. Lucchi, P. Srivastava et al., "Field evaluation of a real-time fluorescence loop-mediated isothermal amplification assay, realamp, for the diagnosis of Malaria in Thailand and India," Journal of Infectious Diseases, vol. 210, no. 8, pp. 11801187, 2014.

[28] V. Jain, A. Agrawal, and N. Singh, "Malaria in a tertiary health care facility of Central India with special reference to severe vivax: Implications for malaria control," Pathogens and Global Health, vol. 107, no. 6, pp. 299-304, 2013.

[29] P. K. Bharti, S. K. Chand, M. P. Singh et al., "Emergence of a new focus of Plasmodium malariae in forest villages of district Balaghat, Central India: Implications for the diagnosis of malaria and its control," Tropical Medicine and International Health, vol. 18, no. 1, pp. 12-17, 2013.

[30] N. Chaturvedi, S. Bhandari, P. K. Bharti, S. K. Basak, M. P. Singh, and N. Singh, "Sympatric distribution of Plasmodium ovale curtisi and P. ovale wallikeri in India: Implication for the diagnosis of malaria and its control," Transactions of the Royal Society of Tropical Medicine and Hygiene, vol. 109, no. 5, pp. 352354,2015

[31] G. Dhangadamajhi, S. K. Kar, and M. R. Ranjit, "High prevalence and gender bias in distribution of Plasmodium malariae infection in central east-coast India," Tropical Biomedicine, vol. 26, no. 3, pp. 326-333, 2009.

[32] N. Kumar, V. Pande, R. M. Bhatt et al., "Genetic deletion of HRP2 and HRP3 in Indian Plasmodium falciparum population and false negative malaria rapid diagnostic test," Acta Tropica, vol. 125, no. 1, pp. 119-121, 2013.

[33] R. K. Tyagi, M. K. Das, S. S. Singh, and Y. D. Sharma, "Discordance in drug resistance-associated mutation patterns in marker genes of plasmodium falciparum and plasmodium knowlesi during coinfections," Journal of Antimicrobial Chemotherapy, vol. 68, no. 5, pp. 1081-1088, 2013.

[34] P. Pati, R. K. Rana, H. K. Khuntia, M. S. Bal, and M. R. Ranjit, "The prvalence of P. malariae in Odisha, India," Tropical Biomedicine, vol. 34, no. 3, pp. 1-8, 2017.

[35] N. K. Shah, G. P. Dhillon, A. P. Dash, U. Arora, S. R. Meshnick, and N. Valecha, "Antimalarial drug resistance of Plasmodium falciparum in India: Changes over time and space," The Lancet Infectious Diseases, vol. 11, no. 1, pp. 57-64, 2011. 
[36] D. D. Laishram, P. L. Sutton, N. Nanda et al., “The complexities of malaria disease manifestations with a focus on asymptomatic malaria," Malaria Journal, vol. 11, article 29, 2012.

[37] U. Shyamsunder Singh, M. Praharaj, C. Sharma, and A. Das, "Paradigm shift in transmission of vector borne diseases," Journal of Emerging Infectious Diseases, vol. 1, no. 4, 2016.

[38] N. Mishra, B. Srivastava, and R. S. Bharti, "Monitoring the efficacy of antimalarial medicines in India via sentinel sites: Outcomes and risk factors for treatment failure," Journal of Vector Borne Diseases, vol. 53, no. 2, p. 168, 2016.

[39] T. Miraclin, A. Matthew, and P. Rupali, "Decreased response to artemisinin combination therapy in falciparum malaria: A preliminary report from South India," Tropical Parasitology, vol. 6, no. 1, p. 85, 2016.

[40] S. K. Ghosh and R. S. Yadav, "Naturally acquired concomitant infections of bancroftian filariasis and human plasmodia in Orissa," Indian Journal of Malariology, vol. 32, no. 1, pp. 32-36, 1995.

[41] P. Jambulingam, S. S. S. Mohapatra, L. K. Das, P. K. Das, and P. K. Rajagopalan, "Detection of Plasmodium ovale in Koraput district, Orissa state," Indian Journal of Medical Research - Section A Infectious Diseases, vol. 89, pp. 115-116, 1989.

[42] M. Zhou, Q. Liu, C. Wongsrichanalai et al., "High prevalence of Plasmodium malariae and Plasmodium ovale in malaria patients along the Thai-Myanmar border, as revealed by acridine orange staining and PCR-based diagnoses," Tropical Medicine and International Health, vol. 3, no. 4, pp. 304-312, 1998.

[43] I. Mueller, P. A. Zimmerman, and J. C. Reeder, "Plasmodium malariae and Plasmodium ovale - the 'bashful' malaria parasites," Trends in Parasitology, vol. 23, no. 6, pp. 278-283, 2007.

[44] P. Li, Z. Zhao, H. Xing et al., "Plasmodium malariae and Plasmodium ovale infections in the China-Myanmar border area," Malaria Journal, vol. 15, no. 1, pp. 557-567, 2016.

[45] K. Maitland, T. N. Williams, and C. I. Newbold, "Plasmodium vivax and P. falciparum: Biological interactions and the possibility of cross-species immunity," Parasitology Today, vol. 13, no. 6, pp. 227-230, 1997.

[46] M. C. Leclerc, J. P. Hugot, P. Durand, and F. Renaud, "Evolutionary relationships between 15 Plasmodium species from New and Old World primates (including humans): An 18S rDNA cladistic analysis," Parasitology, vol. 129, no. 6, pp. 677-684, 2004.

[47] D. H. Kerlin and M. L. Gatton, "Preferential Invasion by Plasmodium Merozoites and the Self-Regulation of Parasite Burden," PLoS ONE, vol. 8, no. 2, Article ID e57434, 2013.

[48] A. Amir, B. Russell, J. W. K. Liew et al., "Invasion characteristics of a Plasmodium knowlesi line newly isolated from a human," Scientific Reports, vol. 6, Article ID 24623, 2016.

[49] R. Poulin, "Interactions between species and the structure of helminth communities," Parasitology, vol. 122, pp. S3-S11, 2001.

[50] C. Lim, E. Hansen, T. M. Desimone et al., "Expansion of host cellular niche can drive adaptation of a zoonotic malaria parasite to humans," Nature Communications, vol. 4, pp. 1638-1656, 2013.

[51] G. G. Rutledge, U. Böhme, M. Sanders et al., "Plasmodium malariae and $\mathrm{P}$. ovale genomes provide insights into malaria parasite evolution," Nature, vol. 542, no. 7639, pp. 101-104, 2017.

[52] X. Y. Yam, T. Brugat, A. Siau et al., "Characterization of the Plasmodium Interspersed Repeats (PIR) proteins of Plasmodium chabaudi indicates functional diversity," Scientific Reports, vol. 6, Article ID 23449, 2016.
[53] A. Fougere, A. P. Jackson, D. P. Bechtsi, J. A. M. Braks, T. Annoura, and J. Fonager, "Variant exported blood-stage proteins encoded by families are expressed in liver stages where they are exported into the parasitophorous vacuole," PLoS Pathogen, vol. 2016, Article ID e1005917, 2016.

[54] S. Nakeesathit, N. Saralamba, S. Pukrittayakamee et al., "Limited polymorphism of the Kelch propeller domain in Plasmodium malariae and P. ovale isolates from Thailand," Antimicrobial Agents and Chemotherapy, vol. 60, no. 7, pp. 4055-4062, 2016.

[55] M. A. Pacheco, F. U. Battistuzzi, R. E. Junge et al., "Timing the origin of human malarias: The lemur puzzle," BMC Evolutionary Biology, vol. 11, no. 1, article 299, 2011.

[56] E. S. Martinsen, S. L. Perkins, and J. J. Schall, "A three-genome phylogeny of malaria parasites (Plasmodium and closely related genera): Evolution of life-history traits and host switches," Molecular Phylogenetics and Evolution, vol. 47, no. 1, pp. 261-273, 2008.

[57] M. C. Bruce, A. Macheso, M. R. Galinski, and J. W. Barnwell, "Characterization and application of multiple genetic markers for Plasmodium malariae," Parasitology, vol. 134, no. 5, pp. 637650, 2007.

[58] J. Westling, C. A. Yowell, P. Majer, J. W. Erickson, J. B. Dame, and B. M. Dunn, "Plasmodium falciparum, P. vivax, and P. malariae: A comparison of the active site properties of plasmepsins cloned and expressed from three different species of the malaria parasite," Experimental Parasitology, vol. 87, no. 3, pp. 185-193, 1997.

[59] A. Das, M. Sharma, B. Gupta, and A. P. Dash, "Plasmodium falciparum and Plasmodium vivax: So similar, yet very different," Parasitology Research, vol. 105, no. 4, pp. 1169-1171, 2009.

[60] J. Richter, G. Franken, H. Mehlhorn, A. Labisch, and D. Häussinger, "What is the evidence for the existence of Plasmodium ovale hypnozoites?" Parasitology Research, vol. 107, no. 6, pp. 1285-1290, 2010.

[61] H. Senn, N. Alattas, A. K. Boggild, and S. K. Morris, "Mixedspecies Plasmodium falciparum and Plasmodium ovale malaria in a paediatric returned traveller," Malaria Journal, vol. 13, no. 1, pp. 78-82, 2014.

[62] M. Rougemont, M. Van Saanen, R. Sahli, H. P. Hinrikson, J. Bille, and K. Jaton, "Detection of four Plasmodium species in blood from humans by $18 \mathrm{~S}$ rRNA gene subunit-based and species-specific real-time PCR assays," Journal of Clinical Microbiology, vol. 42, no. 12, pp. 5636-5643, 2004.

[63] N. Jiang, Q. Chang, X. Sun et al., "Co-infectionswith Plasmodium knowlesi and other Malaria parasites, Myanmar," Emerging Infectious Diseases, vol. 16, no. 9, pp. 1476-1478, 2010.

[64] F. Thomas, K. Mete, S. Helluy et al., "Hitch-hiker parasites or how to benefit from the strategy of another parasite," Evolution, vol. 51, no. 4, pp. 1316-1318, 1997.

[65] M. A. Whittaker, A. J. Dean, and A. Chancellor, "Advocating for malaria elimination - Learning from the successes of other infectious disease elimination programmes," Malaria Journal, vol. 13, no. 1, article 221, 2014.

[66] World Health Organization (WHO), "From malaria control to malaria elimination: a manual for elimination scenario planning," 2014, http://apps.who.int/iris/bitstream/10665/112485/1/ 9789241507028_eng.pdf.

[67] "National Vector-Borne Disease Control Program," 2016, http://nvbdcp.gov.in/Doc/National-framework-for-malaria-elimination-in-India2016\%E2\%80\%932030.pdf. 
[68] D. Menard and A. Dondorp, "Antimalarial Drug Resistance: A Threat to Malaria Elimination," Cold Spring Harbor Perspectives in Medicine, vol. 7, no. 7, Article ID a025619, 2017.

[69] J. L. Waite, S. Swain, P. A. Lynch et al., "Increasing the potential for malaria elimination by targeting zoophilic vectors," Scientific Reports, vol. 7, p. 40551, 2017.

[70] S. P. Sawadogo, A. Niang, E. Bilgo et al., "Targeting male mosquito swarms to control malaria vector density," PLOS ONE, vol. 12, no. 3, Article ID e0173273, 2017. 


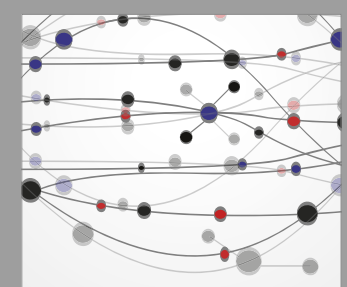

The Scientific World Journal
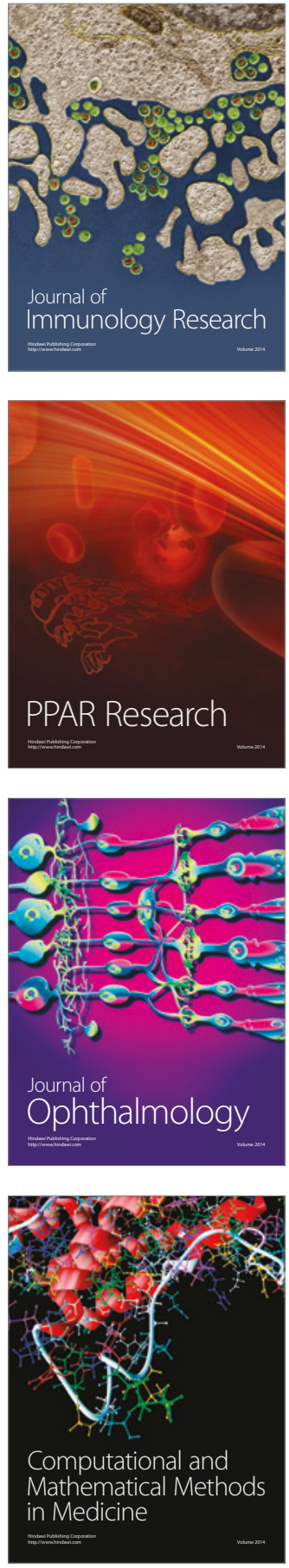

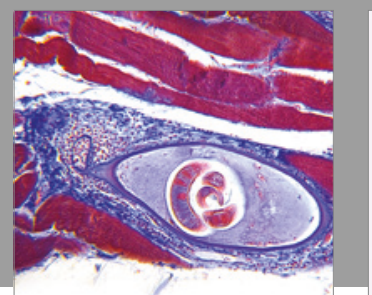

Gastroenterology Research and Practice
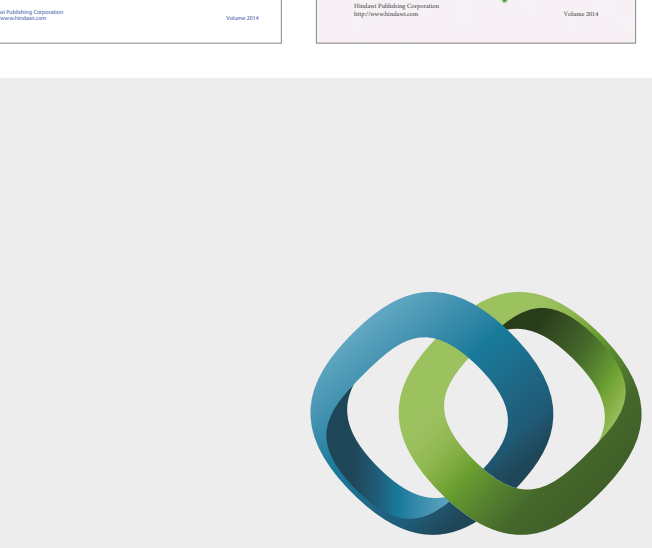

\section{Hindawi}

Submit your manuscripts at

https://www.hindawi.com
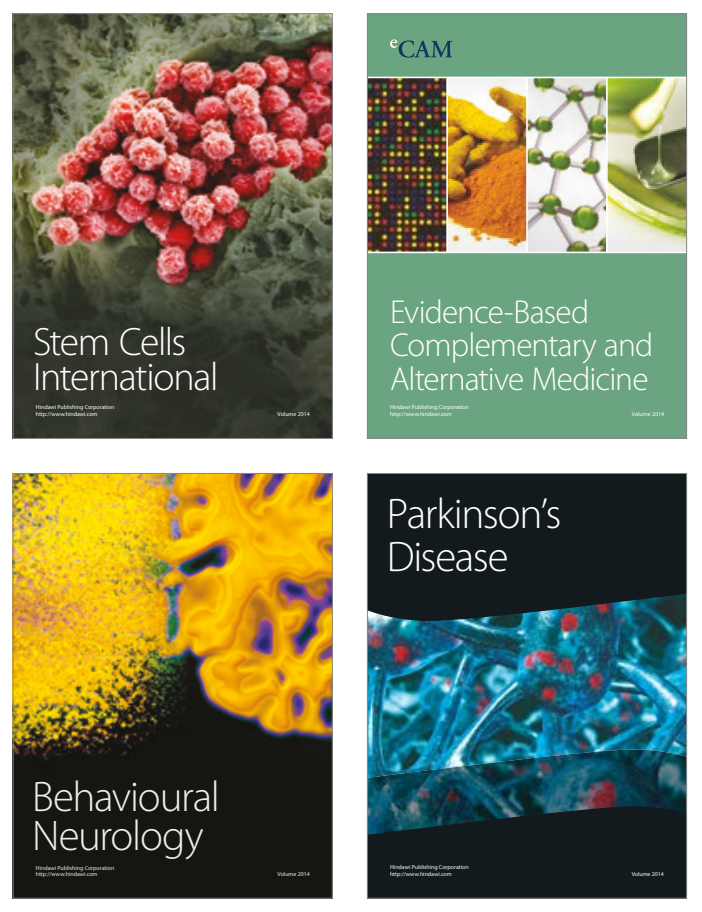
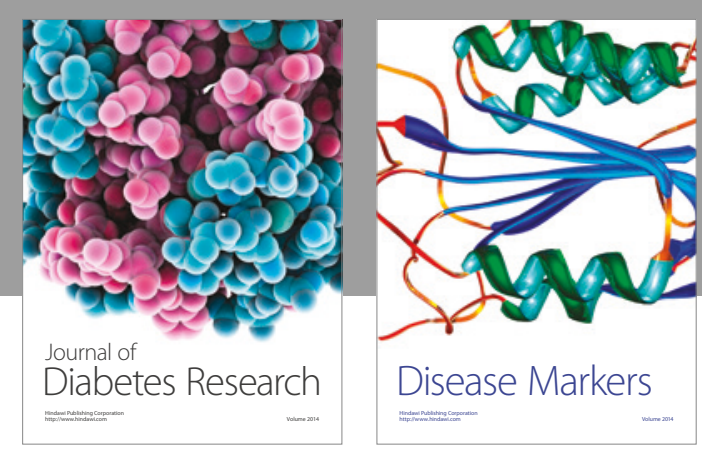

Disease Markers
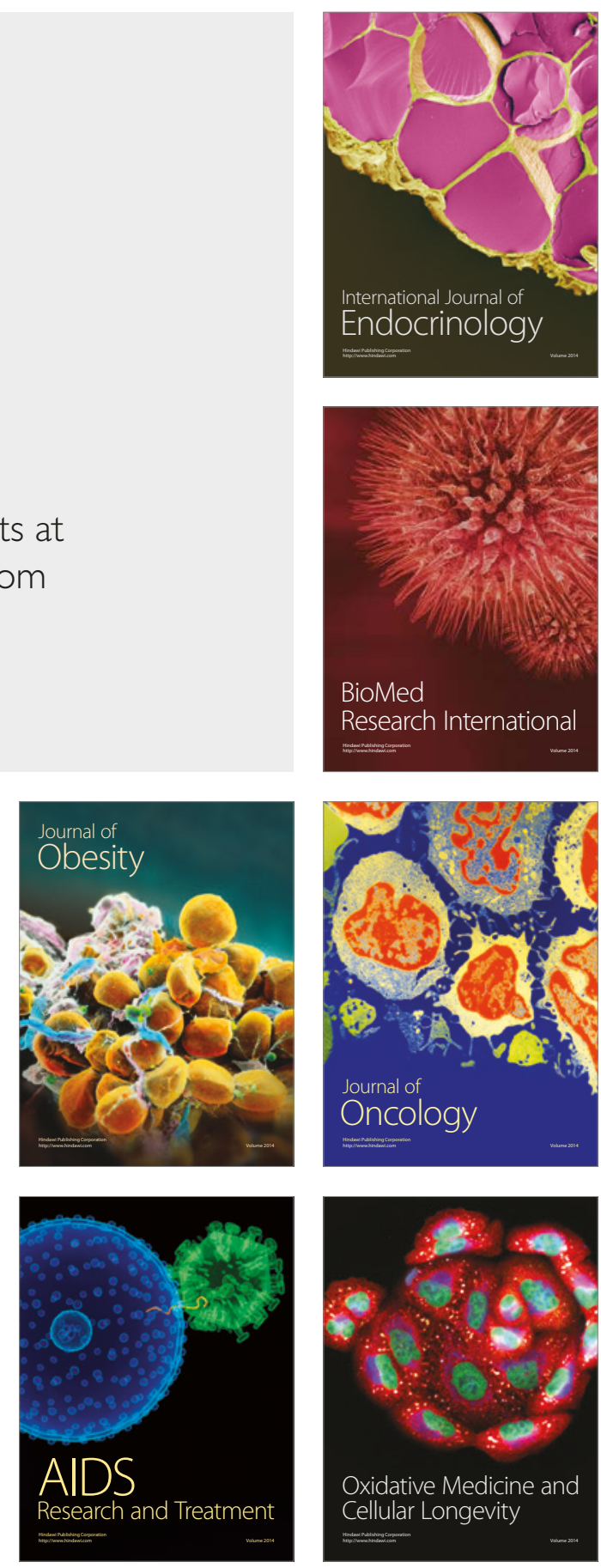